\title{
Decentralize-Change-Recentralize Model of Drug Information Networks in Health Centers: Decentralized Drug Information Services
}

\author{
Dixon Thomas, PhD $^{1}$; Seeba Zachariah, PhD'1 Danial Baker, PharmD, FASCP, FASHP2 \\ ${ }^{1}$ College of Pharmacy, Gulf Medical University, Ajman, UAE \\ ${ }^{2}$ College of Pharmacy and Pharmaceutical Sciences, Washington State University, Spokane, USA.
}

\begin{abstract}
Large health centers usually decentralize their services to small self-sufficient sub-centers of care delivery. These small centers are part of an extensive network of practitioners who are connected. The drug information services of independent clinical pharmacists in a health center could be fragmented. Drug information centers thus need to have a new definition of the mode of operation. While maintaining autonomy in information exchange, professionals are integrated to form a large community of practitioners. Technological advancements in communication and access to resources enable efficient collaborations to happen. Immersed in patient-centered collaborative practice environments, networks of professionals integrate drug information services. Drug information networks thus hold a similar philosophy of health centers to decentralize-change-centralize its services. Further research is required to measure the impact of this model of drug information services.
\end{abstract}

Keywords: Decentralization, drug information, clinical pharmacy, health centers

The decentralization of healthcare services is a trend globally. Decentralization in health systems involves moving decision making away from centralized control and closer to the users of health services. ${ }^{1}$ Hospital pharmacy services have been decentralized for many years and its effectiveness needs to be studied further. Decentralizing pharmacy services was found to improve patient safety. Clinical pharmacists with autonomy provide many services, including pharmacovigilance and drug information at bedside. ${ }^{2}$ This decentralization decreased questions reaching drug information centers as clinical pharmacists at practice sites answer them. Improved accessibility to drug information resources and training on how to use them enables pharmacists to practice evidence-based. Pharmacy practice in different parts of healthcare institutions is collaborative, patient-centered, and evidence-based. Education, practice and research are essential operations of such institutions. Such large academic health centers have networks of small practice groups working towards achieving a joint mission. They have great potential for data sharing to create and use evidence for better practice. ${ }^{3}$ Even though the environment is favoring integrated decentralized drug information services, lack of coordination shall result in fragmented or inconsistent services.

Corresponding author: Dixon Thomas, PhD

Associate Professor \& Chair, Dept. of Pharmacy Practice

College of Pharmacy, Gulf Medical University, UAE

+971-557540701; dr.dixon@gmu.ac.ae

dixon.thomas@gmail.com
The transformation that happened to drug information centers could be shortly represented as decentralize-changerecentralize, which is based on Kurt Lewin's famous change management model commonly framed now as unfreezechange-refreeze. ${ }^{4}$ Just having a conceptual model is not enough for effective change. Increased attention to the change process is essential for effective implementation and results, depending on the context it is implemented..$^{5}$ Decentralization might impact health system equity, efficiency and resilience. The contextual factors are to be considered in maximizing positive effects and minimizing the negative effects of decentralization. ${ }^{6}$ Coordination of smaller units of information management results in recentralizing the efforts to a large network of professionals. The impact of decentralization of drug information services is not well demonstrated in the literature. Instead of proper decentralization, drug information service might have been neglected as a vital service of pharmacists.

Drug information centers were established as centralized services that patients and health professionals could approach. There is a gradual decline in the number of such centers and access to similar information became more widely available at other locations (e.g., all pharmacy locations, nursing stations) in health centers. ${ }^{7}$ Many of these drug information centers were closed and others restrict their services mostly to formulary management and patient safety-related initiatives. ${ }^{8}$ It became hard to justify the return of investment on human resources and the acquisition and subscription cost of these centrally held drug information resources. It was then necessary to decrease the staffing of these drug information centers and find ways to make subscribed resources accessible directly to practitioners and students within academic and other health centers. 
Over the past few decades, pharmacy services, in general, became more decentralized in hospital settings. Both clinical and distribution services were decentralized to be more feasible, efficient and cost-effective. ${ }^{9}$ Pharmacists supported this decentralization effort to evidence-based, patientcentered practice and interprofessional care provided through clinical services. The drug information skills of pharmacists are helpful in providing evidence-based pharmacy services in direct patient care areas. ${ }^{10}$

The networking of pharmacists could improve drug information services by recentralizing the efforts of maintaining autonomy at the workplace. Sharing of resources and expertise is helpful in better clinical decision making. Figure 1, a network of decentralized drug information centers illustrates how decentralization of drug information services is integrated into large health centers. In practice, many pharmacists could provide drug information services in isolation resulting in a fragmented system of practice. Fragmented drug information could be inconsistent in providing the best quality information. Having a network of professionals will improve the capacity of the whole system. Share of resources facilitates evidence-based practice. These networks could be connected to professionals outside the institution too. Such networks motivate professionals to expand their services. It increases more recognition to the experts broadening their information support. Proper decentralization of drug information services promotes more benefits.

\section{Network of decentralized drug information services}

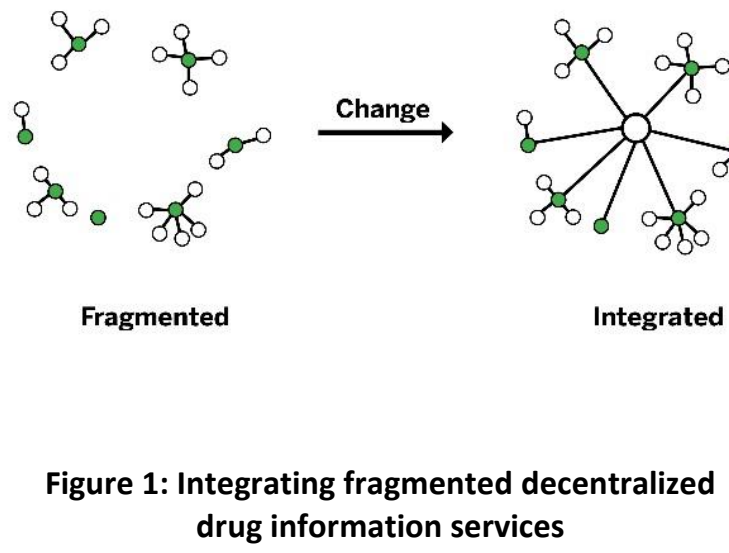

Decrease the workload of the center by self-sufficient pharmacists providing drug information in the area of their expertise with evidence support and considering patient values. Pharmacists at their workplaces are in advantage of close contact with health professionals and patients they are dealing with to provide contextually appropriate drug information. These services are to individualize patient care with patientspecific drug information.
Drug information centers focus on standardizing drug use by assisting in clinical practice guidelines, including pharmacovigilance, pharmacokinetics, pharmacogenomics, pharmacoeconomics, and treatment effectiveness data pooled with central intelligence and external referencing. The centers could also focus on preparing drug monographs to manage drug formulary with informed decision-making. Drug information service stays as a medium of communication between different stakeholders and pharmacists.

A technology-enabled network of professionals within the center and outside help share expertise even internationally. One such example is intravenous parenteral nutrition (IVPN) network managed by Cleveland Clinic Abu Dhabi. Drug information centers thus become a center of centers or a network of networks.

Providing access to drug information resources, any professionals could obtain information and consult with a pharmacist when needed. The center could conduct training programs to enable pharmacists and other health professionals to use drug information centers. This could also include integrating drug information resources to the hospital information management system.

Decentralized drug information centers are efficient systems in need of limited funds on their own as no individual needs to be employed just for this purpose and no information resource to be purchased for itself. It is a network of professionals appointed to perform their duties that generate income and valuable patient outcomes. Thus, the drug information center is well integrated to strengthen bonds of health centers with no significant increase in financial burden. It solves the sustainability issue of the old centralized model of drug information services.

The decentralized drug information services use existing communication systems. This resulted in more practitioners have access to good quality information. Most drug information resources transformed into an easy to find, read, and apply format. With even artificial intelligence, some of these resources are being integrated into health information databases and the electronic medical record and prescribing platforms. Clinician training has also evolved and incorporated this type of education into their formal education and practicebased training programs. Most health professions education trains their students to be ready to practice evidence-based. Also, health regulators have mandated that practitioners receive continuing education to use current best quality evidence in their practice. Professional agencies created clinical practice guidelines using evidence-based practice approaches instead of just expert opinion methods. Despite these changes in access and education, there is still a gap between new research evidence and changes in practice. ${ }^{11}$ Drug information service fosters evidence-based practice. 
Patient-centeredness improved patient access to drug information. ${ }^{12}$ Treating health professionals helped in the interpretation of information and guided patient decision making. Patient medication adherence is expected to improve when patients are involved in clinical decision making before the writing of prescriptions. Patient-focused communication is an essential skill for all practitioners to make this type of impact. $^{13}$

Interprofessional care allows for the perspectives of different professions to be considered. This type of collaboration should result in improved patient care and further reinforcement of the value of professional collaboration. Educating future health professionals when they are still students to collaborate and socialize should develop a better sense of how health professions contributes to patient care. Interprofessional education aids the formation of more cooperative interprofessional community. ${ }^{14}$ Cooperation of professionals and patients creates a platform for drug information exchange.

Drug information centers have a new definition of space and approach of practice. The drug information center has a combination of actual and virtual spaces with an extensive network of professionals within and outside an individual institution. The physical space present is just a station from where to coordinate operations. The approach of practice is to promote its operations from natural locations of patient care. Professionals collaborating with the patient in the care process are empowered and guided to be self-sufficient. ${ }^{15}$

Practitioners, while belonging to a specialized area of work, have a sense of belonging to a macro network of drug information stakeholders, still called a center at large. Large healthcare institutions with academic, clinical, and research operations are now being connected as academic health centers. ${ }^{16}$ Drug information centers have a broad scope in such academic health centers through its integrated networks. Being part of an academic and research institution is favorable for evidence-based practice because of its focus and mission. As we went far to fragmented decentralized drug information services, recentralizing partially could help in mending the bonds. The model decentralize-change-recentralize explains how proper decentralization of drug information services improves capacity and coordinate the efforts to support each other. The impact of such pharmacist-led drug information networks in health centers is to be studied on patient outcomes at large. Implementation of evidence-based pharmacy on a large scale should improve public trust.

Funding information: No funds received.

Conflicts of interest: None.

Acknowledgement: None.

Ethics approval: Not required.

\section{References}

1. Saltman BS, Bankauskaite V, Vrangbaek K, World Health Organization. Regional Office for Europe \& European Observatory on Health Systems and Policies. Decentralization in Health Care: Strategies and Outcomes. Open University Press; 2007. pp. 1-6. https://apps.who.int/iris/handle/10665/107816

2. Thomas D, Marriott J, Vadlamudi R, Efendie B, Maine LL. Introduction to Clinical Practice, Research, and Pharmacy Education. Thomas D (ed) Clinical Pharmacy Education, Practice and Research: Clinical Pharmacy, Drug Information, Pharmacovigilance, Pharmacoeconomics and Clinical Research. Elsevier; 2019. pp. 1-9. https://doi.org/10.1016/B978-0-12814276-9.00001-5

3. Piwowar HA, Becich MJ, Bilofsky H, Crowley RS. Towards a data sharing culture: recommendations for leadership from academic health centers. PLoS Medicine. 2008 Sep;5(9). https://doi.org/10.1371/journal.pmed.0050183

4. Cummings $S$, Bridgman T, Brown KG. Unfreezing change as three steps: Rethinking Kurt Lewin's legacy for change management. Human Relations. 2016 Jan;69(1):33-60. https://doi.org/10.1177/0018726715577707

5. Dickson G, Lindstrom R, Black C, Van der Gucht D. Evidence-informed change management in Canadian healthcare organizations. Ottawa: Canadian Health Services Research Foundation. 2012 Jun. https://www.cfhi-fcass.ca/sf-docs/defaultsource/commissioned-research-reports/dicksonen.pdf. Accessed: 29 January 2020.

6. Abimbola S, Baatiema L, Bigdeli M. The impacts of decentralization on health system equity, efficiency and resilience: a realist synthesis of the evidence. Health Policy and Planning. 2019 Oct 1;34(8):605-17. https://doi.org/10.1093/heapol/czz055

7. Rosenberg JM, Schilit S, Nathan JP, Zerilli T, Mcguire $\mathrm{H}$. Update on the status of 89 drug information centers in the United States. American Journal of Health-System Pharmacy. 2009 Oct 1;66(19):1718-22. https://doi.org/10.2146/ajhp080563

8. Gabay MP. The evolution of drug information centers and specialists. Hosp Pharm. 2017 Jul; 52(7): 452453. https://doi.org/10.1177/0018578717724235

9. Pro $M L$, Pro $A M$, Pro DS, Pro AT, Pro DD, Con MH, Con TS, Con TM, Con GS, Con SL. Are decentralized pharmacy services the preferred model of pharmacy service delivery within a hospital? The Canadian Journal of Hospital Pharmacy. 2015 Apr 28;68(2): 168-171. http://dx.doi.org/10.4212/cjhp.v68i2.1444 
10. Jacobi J, Ray S, Danelich I, Dodds Ashley E, Eckel S, Guharoy R, Militello M, O'Donnell P, Sam T, Crist SM, Smidt D. Impact of the pharmacy practice model initiative on clinical pharmacy specialist practice. Pharmacotherapy: The Journal of Human Pharmacology and Drug Therapy. 2016 May;36(5):e40-9. http://doi:10.1002/phar.1745

11. Kristensen $\mathrm{N}$, Nymann $\mathrm{C}$, Konradsen $\mathrm{H}$. Implementing research results in clinical practice-the experiences of healthcare professionals. BMC Health Services Research. 2015 Dec;16(1):48. https://doi.org/10.1186/s12913-016-1292-y

12. Prey JE, Restaino S, Vawdrey DK. Providing hospital patients with access to their medical records. AMIA Annual Symposium Proc. 2014; 2014: 1884-1893.

13. Lee SW, Thomas D, Zachariah S, Cooper JC. Communication Skills and Patient History Interview. Thomas D (ed) Clinical Pharmacy Education, Practice and Research: Clinical Pharmacy, Drug Information, Pharmacovigilance, Pharmacoeconomics and Clinical Research. Elsevier; 2019. pp. 79-89. https://doi.org/10.1016/B978-0-12-814276-9.00006$\underline{4}$

14. Joynes VC. Defining and understanding the relationship between professional identity and interprofessional responsibility: implications for educating health and social care students. Advances in Health Sciences Education. 2018 Mar 1;23(1):13349. http://doi:10.1007/s10459-017-9778-x

15. Castro EM, Van Regenmortel T, Vanhaecht K, Sermeus W, Van Hecke A. Patient empowerment, patient participation and patient-centeredness in hospital care: A concept analysis based on a literature review. Patient Education and Counseling. 2016 Dec 1;99(12):1923-39. https://doi.org/10.1016/j.pec.2016.07.026

16. Johnston SC. Academic medical centers: too large for their own health? JAMA. 2019;322(3):203-204. https://doi:10.1001/jama.2019.6834 